\title{
Robust Fixed Point Transformation Based Design for Model Reference Adaptive Control of a Modified TORA System
}

\author{
József K. Tar, Teréz A. Várkonyi, Levente Kovács, Imre J. Rudas and Tamás Haidegger
}

\begin{abstract}
Benchmark problems continue to represent an actively studied domain, focusing on application-based situations, where controllers have to deal with typical real environments. In this paper, a Robust Fixed Point Transformations (RFPT)-based Model Reference Adaptive Controller (MRAC) is designed for a modified Translational Oscillations by a Rotational Actuator (TORA) system, which is an indirectly driven, underactuated classical mechanical system with peculiar properties. The RFPT-based design has the advantage of working only with three free parameters, and does not need complex $a$ priori calculations. It is founded on the idea that at the cost of replacing the requirement for global stability with local stability, a mathematically very simple and geometrically lucid, well interpreted methodology can be developed. The resulting structure directly concentrates on the primary design intent, i.e., on the realization of a purely kinematically prescribed trajectory tracking. Examples and simulation results are presented in this paper, demonstrating that the RFPT-based design can provide an efficient MRAC controller for a very special physical system.
\end{abstract}

\section{INTRODUCTION}

In the field of control engineering, benchmark problems are usually easy to be modeled, and represent typical problems controllers have to deal with in real applications (underactuation, singularities, etc.). The main purpose is to reveal the strengths and weaknesses of the controllers, and to compare control solutions to each other. Plenty of benchmark examples can be found in the literature, e.g., control of missile autopilot (aero) [1], hydraulic positioning system (servo) [2], inverted pendulum (mechanical) [3] and the Translational Oscillations by a Rotational Actuator (TORA) system [4].

Originally, the TORA system was considered as a simplified model of a dual-spin spacecraft with mass imbalance. The system is indirectly driven, underactuated, and contains dynamic singularities, which makes it a relevant benchmark problem, since real dynamic systems-almost alwayspresent at least one of these attributes. Many examples can be found in the literature of controlling the TORA system, e.g., the design of cascade and passivity based controllers [5], [6], or the application of the Tensor Product Form' of the system model, applied to develop a model-based controller [7].

There are different aspects based on which adaptive controllers can be designed. One of the most popular approaches is global stability for its trustworthiness, which can be realized with Lyapunov's method. The key point is to elaborate

\footnotetext{
${ }^{1}$ Antal Bejczy Center for Intelligent Robotics, Óbuda University, Budapest, Hungary \{tar.jozsef@nik., teri.varkonyi@nik., kovacs.levente@nik., rudase, haideggere\}uni-obuda.hu
}

an appropriate Lyapunov function from an initial "candidate", and prove its non-positive time-derivative. Despite the fact that Lyapunov's method guarantees global stability, unfortunately, it cannot be derived automatically, and relies on a lot of parameters. Thus, in many cases, it needs high mathematical skills and complex calculations. Nevertheless, due to its trustworthiness and ingeniousness, most of the controllers are designed by Lyapunov's method, e.g., the Slotine-Li controller [8], most of the adaptive controllers [9], some of the adaptive robust controller [10] and numerous of the robot controllers [11].

Within the class of adaptive controllers, Model Reference Adaptive Controller (MRAC) is specially interesting since it is transparent and can be handled easily. While the main task of the whole controller is to guarantee precise trajectory tracking, it has a special property generating an illusion that it controls a system, having the dynamic properties of a "reference model". The MRAC controllers have become very popular in various applications, e.g., for the dynamic control of a Stewart platform [12], for implementing impedance control for robots [13], in robotics applications in general [14] and in teleoperation systems [15].

Another aspect in designing controllers is to be automated and interpreted in a lucid geometrical way. An example for this is the method called Robust Fixed Point Transformations (RFPT). This method normally generates contractive maps in Banach Spaces by the use of saturated sigmoid functions. Such a map is defined over the observable realized response and the desired response of the controlled system, and generates an iterative sequence of control signals. This sequence converges to the solution of the control task. Unfortunately, the convergence holds only within a bounded region that the system in principle can leave. Because of this, no global stability can be guaranteed in this manner. Fortunately, from practical point of view, global stability is not always necessary for trustworthy control, even the operation of modern robust controllers is guaranteed only with limitations [16]. For this reason, the need for global (global asymptotic) stability can be dropped.

In this paper, the operation of an RFPT-based Model Reference Adaptive Controller is investigated for the control of the modified TORA system which is a classical mechanical system having peculiar properties. The results show that though the modified TORA system is indirectly driven, strongly underactuated and contains singularities, the RFPTbased controller can work well in the control process.

The rest of the paper is organized as follows: in Section II, the possible typical divergences of the RFPT-based con- 
trollers are briefed. Section III describes the dynamic model of the modified TORA system and Section IV provides the simulation results. Finally, Section V gives the conclusions.

\section{Possible Divergences of the RFPT-BASED CONTROLlERS}

Below, the basics and the possible divergences of Robust Fixed Point Transformations are summarized. The RFPTbased design assumes that we are in the possession of a rough dynamic model of the system under control. By the use of the model, the control signal $Q^{D e s}$ can be determined for a purely kinematically designed desired system response $r^{D e s}$ in a given, known state $[q, \dot{q}]$ of the system:

$$
Q^{\text {Des }}=\Phi^{\text {Rough }}\left(q, \dot{q}, r^{\text {Des }}\right) .
$$

Normally, the desired response may correspond to the desired $2^{\text {nd }}$ time-derivatives of the generalized coordinates $q$ of the classical mechanical systems: $r^{D e s}=\ddot{q}^{D e s}$ and $Q^{D e s}=$ $\Phi^{\text {Rough }}\left(q, \dot{q}, \ddot{q}^{D e s}\right)$. The realized response $r$ (that is assumed to be directly observable) depends on $q, \dot{q}, Q^{D e s}$ and on the external disturbances $Q^{\text {Dist }}$, since

$$
r:=\Psi^{\text {Exact }}\left(q, \dot{q}, Q^{\text {Des }}, Q^{\text {Dist }}\right),
$$

where $\Psi^{\text {Exact }}$ denotes the real system with disturbances. The available model, $\Phi^{\text {Rough }}$ is normally incomplete and imprecise: $r \neq r^{D e s}$, even if the external disturbance is lacking. Let us denote $n$ the number of the control cycles, then the same can be stated for every control cycle, and $r_{n} \neq r_{n}^{\text {Des }}$ will occur.

In [17], for a "Single Input - Single Output (SISO)" system, an RFPT-based design for MRAC controllers is suggested. Without any further mathematical considerations, the "Reference Model" can take the role of the "Rough Model", and instead of applying directly the above shown control force $Q_{n}^{\text {Des }}$, iterative control signals are used:

$$
Q_{n+1}^{D e f}=G\left(Q_{n}^{D e f}, f_{n}, Q_{n+1}^{D e s}\right),
$$

as

$$
\begin{gathered}
G\left(Q_{n}^{D e f}, f_{n}, Q_{n+1}^{D e s}\right):= \\
\left(Q_{n}^{D e f}+K_{c}\right)\left(1+B_{c} \sigma\left(A_{c}\left[f_{n}-Q_{n+1}^{D e s}\right]\right)\right)-K_{c},
\end{gathered}
$$

where $Q^{\text {Def }}$ denotes the adaptive deformation of $Q^{\text {Des }}$, $f_{n}=\Phi^{\text {Rough }}\left(r_{n}\right), \sigma(x) \in(-1,+1)$ is a monotone increasing sigmoid function with the property $\sigma(0)=0$ and derivative $\sigma^{\prime}(0)=1, B_{c}= \pm 1$, and $K_{c}$ and $A_{c}$ are adaptive control parameters.

Observe that if $f_{n}=Q_{n+1}^{D e s}$, then $r_{n}=r_{n+1}^{D e s}$. In this case, $Q_{n+1}^{D e f}=G\left(Q_{n+1}^{D e f}, f_{n}, Q_{n+1}^{D e s}\right)$ which means that the solution of the control task is the fixed point of the mapping, defined by function $G$ that substantiates the name of the method. It is straightforward, too, that $G\left(-K_{c}, f_{n}, Q_{n+1}^{D e s}\right)=-K_{c}$, i.e., $-K_{c}$ is a trivial fixed point of this function. Therefore our duty is to find a setting for the parameters $K_{c}, B_{c}$ and $A_{c}$, at which the solution is an attractive, and $-K_{c}$ is a repulsive fixed point of the iteration. In this case, the iterative sequence will converge to the solution within its basin of attraction. It is well known from [18] that a fixed point is attractive, if the function is almost flat around it.

The behavior of the sequence $\left\{Q_{n}^{D e f}\right\}$ outside the region of convergence depends on the global properties of $G$. In [19], a chattering-like behavior was accidentally found and was further analyzed in [20]. A more detailed analysis in [21] revealed that the following cases may happen:

a) monotone or non-monotone convergence to the solution of the control task,

b) bounded chaotic behavior that can be treated by the method outlined in [19],

c) divergence to infinity,

d) convergence to the trivial fixed point of $G$ i.e., to $-K_{c}$.

It was also shown in [21] that in the vicinity of the nontrivial fixed point, appropriate fixed $K_{c}$ and $B_{c}$ parameters can be chosen at which for very small $A_{c}$, the convergence is monotone, and with increasing $A_{c}$, this iteration turns to fluctuating before becoming bounded chaotic. These small fluctuations are called "precursor oscillations". It was shown that by constructing a model-independent observer for such fluctuations, and by tuning $A_{c}$ the fluctuation can be kept under efficient control and the controller can be kept in the convergent region. With this improvement, the RFPT-based design has become "competitive" with the more complicated Lyapunov function-based methods.

\section{Modeling Methods}

In this section, the dynamic model of our TORA system in introduced. The schematic view of the system is shown in Fig. 1. The original system consists of a cart, the linear motion of which is controlled by a driven rotational axle moving a pendulum as a counterbalance. The system is "indirectly driven", since there is no direct driving component in the "horizontal" direction: the center of mass of the whole system cannot be accelerated with respect to an inertial system of reference. Consequently, any rotation of the axle of the pendulum modifies the mass distribution of the whole system, therefore the position of the cart has to vary, too. This system is "underactuated" as well, due to the fact that for its 2 Degrees of Freedom ( $D o F$ ) (a rotational and a linear), it has only one directly driven axle. The system also has a spring that limits its horizontal motion that could be unlimited in the case of constant velocity.

In our example, the system is modified: the horizontal spring is lacking, and the presence of gravitational acceleration is assumed, which qualifies it to be considered as a benchmark problem, too, for designing an adaptive controller. By removing the spring, this modified system is curtailed of its "horizontal" stabilizing mechanism, consequently its precise control means a greater challenge than that of the original TORA. Besides, the application of the gravitational term introduces an extra non-linearity, that further complicates its control.

The equations of motion of the indirectly driven underactuated 2 DoF system are: 


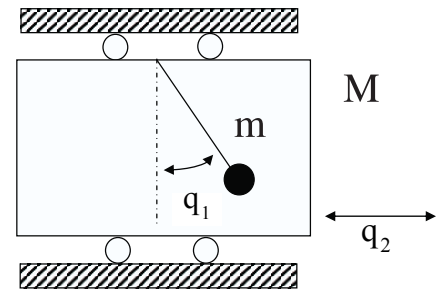

Fig. 1. Model of the TORA system, where $q_{1}$ is the rotation angle of the pendulum, $q_{2}$ denotes the linear displacement of the cart's body, $m$ stands for the mass of the point-like counterweight and $M$ marks the mass of the body of the cart.

$$
\begin{gathered}
{\left[\begin{array}{cc}
m L^{2} & m L \cos \left(q_{1}\right) \\
m L \cos \left(q_{1}\right) & m+M
\end{array}\right]\left[\begin{array}{l}
\ddot{q}_{1} \\
\ddot{q}_{2}
\end{array}\right]+} \\
+\left[\begin{array}{c}
-m L g \sin \left(q_{1}\right) \\
-m L \sin \left(q_{1}\right) \dot{q}_{1}^{2}
\end{array}\right]=\left[\begin{array}{l}
Q_{1} \\
Q_{2}
\end{array}\right]
\end{gathered}
$$

Its generalized coordinates are $q_{1}[\mathrm{rad}]$ (rotation angle of the pendulum), and $q_{2}[\mathrm{~m}]$ (linear displacement of the cart's body). The dynamic parameters of the controlled system are as follows: $m=20[\mathrm{~kg}]$ (the mass of the point-like counterweight), $M=30[\mathrm{~kg}]$ (the mass of the body of the cart, $L=2[\mathrm{~m}]$ (the length of the beam).

The reference system has the same kinematic structure but the parameters are different (the difference is approximately under $25 \%$, the values are randomly chosen): $\tilde{m}=15[\mathrm{~kg}]$, $\tilde{M}=25[\mathrm{~kg}]$ and $\tilde{L}=2[\mathrm{~m}]$ (the same). The gravitational acceleration is assumed to be $g=10 \mathrm{~m} / \mathrm{s}^{2}$. The generalized forces to be exerted by the controller are $Q_{1}[N \times m]$ (torque at axle 1) and $Q_{2} \equiv 0[N]$ (force pushing the cart in the lateral direction; since our system is indirect and underactuated driving system, it has to be identical to zero).

\section{Simulation RESUlTS}

In this section, the control method and some representative simulation results are briefly summarized.

\section{A. The control method}

The above mentioned RFPT-based MRAC controller is applied (see Fig. 2). Eq. (5) can be used in the following manner: first of all, a nominal motion $q_{2}^{N o m}(t)$ is determined for the system. After that, a kinematically defined PID-type controller determines the "desired" (corrected) acceleration, $\ddot{q}_{2}^{D e s}(t)$, based on the nominal motion and the previous system response. By substituting $\ddot{q}_{2}^{D e s}(t)$ into Eq. (5) (and by using that $\left.Q_{2}=0\right), \ddot{q}_{1}^{D e s}(t)$ can be calculated as

$$
\ddot{q}_{1}^{D e s}=\frac{m L \sin \left(q_{1}\right) \dot{q}_{1}^{2}-(M+m) \ddot{q}_{2}^{D e s}}{m L \cos \left(q_{1}\right)} .
$$

Via substituting $\ddot{q}_{2}^{D e s}$ and $\ddot{q}_{1}^{\text {Des }}$ into the first line of (5), the following peculiar equation of motion is obtained:

$$
\begin{gathered}
\frac{m L \cos ^{2} q_{1}-(m+M) L}{\cos q_{1}} \ddot{q}_{2}^{D e s}+ \\
m L^{2} \tan q_{1} \dot{q}_{1}^{2}-m L g \sin q_{1}=Q_{1}
\end{gathered}
$$

where the "inertia term" can be rewritten as:

$$
\begin{gathered}
L \frac{\left(\cos ^{2} q_{1}-1\right) m-M}{\cos q_{1}}, \text { in which } \\
0>\left(\cos ^{2} q_{1}-1\right) m-M \in[-m-M,-M] .
\end{gathered}
$$

It may have varying sign and evidently, it is singular at $q_{1}= \pm \frac{\pi}{2}$, therefore, dynamic singularity is introduced into the indirectly driven system.

By substituting these values into the first line of the equations of motion of the reference system (the same equation as (7) but it contains the parameters of the reference system), the torque $Q_{1}^{D e s}$ can be determined.

In the next step, the RFPT-based MRAC controller makes an iteration with function $G$ (see Eq. (4)). Finally, the adaptively deformed $Q_{1}^{D e f}$ value is exerted on the controlled system in the system state $(q, \dot{q})$, and the response of the controlled system $\ddot{q}$ is obtained. These values can be substituted into the reference model to obtain the recalculated force need of the reference system for the actual system response marked by $f_{n}$ in Section II and marked by $Q_{1}^{\text {Sim }}$ in the simulation results. If the controller is convergent then the following situation occurs:

$$
Q_{1}^{\text {Des }} \rightarrow Q_{1}^{\text {Sim }} \neq Q_{1}^{\text {Def }}, \text { while } \ddot{q}_{2} \rightarrow \ddot{q}_{2}^{\text {Des }}
$$

This corresponds to the "MRAC illusion": the controller calculates with kinematically prescribed $2^{\text {nd }}$ time-derivatives and the dynamics of the reference model, thus obtains precise tracking.

In the examples, the PID-type kinematic tracking is prescribed for the system by determining an exponential decay rate for the tracking error with exponent $\Lambda>0$ in the form $\delta q(t) \propto \delta q\left(t_{0}\right) e^{-\Lambda\left(t-t_{0}\right)}$. By manipulating the integrated tracking error as $\left(\frac{d}{d t}+\Lambda\right)^{3} \int_{t_{0}}^{t}\left(q^{N}(\xi)-q(\xi)\right) d \xi \equiv 0$ the following can be gained:

$$
\begin{gathered}
\ddot{q}_{2}^{\text {Des }}=\ddot{q}_{2}^{N o m}+3 \Lambda^{2}\left(q_{2}^{N o m}-q_{2}\right)+3 \Lambda\left(\dot{q}_{2}^{N o m}-\dot{q}_{2}\right)+ \\
+\Lambda^{3} \int_{0}^{t}\left(q_{2}^{N o m}(\tau)-q_{2}(\tau)\right) d \tau
\end{gathered}
$$

The appropriate value for $\Lambda$ is determined by the dynamic details of the trajectory to be tracked. For tracking a signal of maximal significant frequency component $\Omega$, trajectory tracking will reveal the fine details of the nominal motion if $\Lambda \gg \omega$. In our case, for nominal trajectory, a $3^{\text {rd }}$ order spline function of time with the period of $6 s$ multiplied by a $\tanh (\Omega t)$-type "moderator" that guarantees initial conditions $q_{i n i}^{N o m}=0$ and $\dot{q}_{i n i}^{N o m}=0$ was chosen in which $\ddot{q}_{2}^{\text {Nom }}$ linearly varies within certain time-intervals and its timederivative abruptly changes at their boundaries. This trajectory has far richer dynamics than a constant set value to be achieved. In our case, the choice of $\Lambda=12 / s$ (for $\Omega=6 H z$ ) was satisfactory for tracking these trajectories. Since the initial state of the system is rest, the differences in the initial states of the nominal and the simulated trajectories are zero. In this manner the occurrence of rough and drastic initial transients can be avoided. For the details of the nominal trajectory see Fig. 3. 


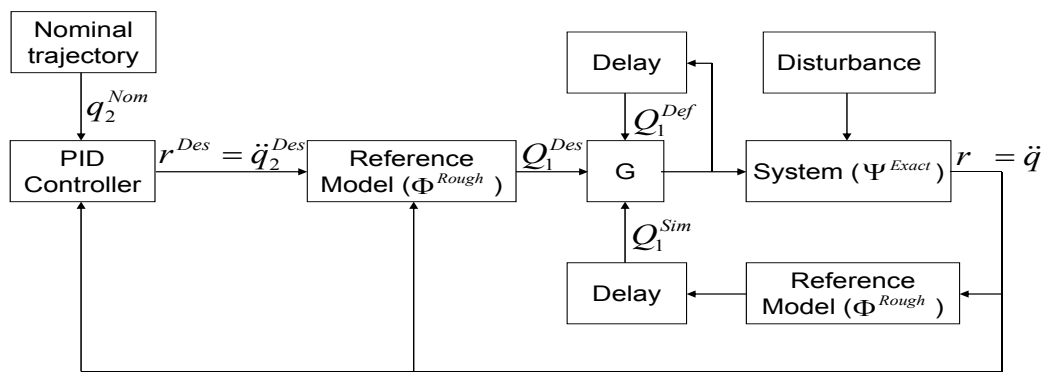

Fig. 2. The RFPT-based MRAC control scheme.

The adaptive control parameters are $K_{c}=10^{5}, B_{c}=-1$ and $A_{c}$ is tuned from its minimal value $10^{-5}$. The cycle time of the digital controller is assumed to be $\Delta t:=t_{n}-$ $t_{n-1}=10^{-3}[\mathrm{~s}]$. To approximate the operation of this digital controller there are two different ways: we can either keep constant forces during the intervals $\left[t_{n-1}, t_{n}\right]$, or (for a more speedy approach for the numerical calculations) we can insist on maintaining fixed delay time $\Delta t$ between the "actual" and the "delayed" inputs of function $G$ in (4). The whole problem can be modeled as a system of delayed nonlinear differential equations. In this case, the time-resolution of the numerical integration can be different of the cycle time, and the cyclic nature of the controller is taken into account by properly dealing with the delayed signals. Results of ample simulation tests made in the past have justified that this approach yields acceptable results. In the forthcoming simulations this approach is chosen with a step length of integration freely determined by the simulator.

The observer developed to avoid chattering and torque fluctuation calculates the scalar product defined for vectorial force components (also valid for single component vectors) as:

$$
\begin{gathered}
F\left(t_{n}\right):=\left(\vec{Q}\left(t_{n}\right)-\vec{Q}\left(t_{n-1}\right)\right)^{T} \times \\
\times\left(\vec{Q}\left(t_{n-1}\right)-\vec{Q}\left(t_{n-2}\right)\right) .
\end{gathered}
$$

If the variation of the consecutive force terms is approximately in the same direction, then these scalar products are positive. In the case of fluctuations between the consecutive cycles, these variations are approximately in the opposite direction, therefore the scalar products are negative. If these contributions are stored in a buffer by a forgetting integral with output:

$$
\hat{F}_{n}:=(1-\beta) \sum_{s=0}^{n} \beta^{s} F_{n-s},
$$

where $\beta \in(0,1)$ then in the fluctuation-free sessions, values $\hat{F}_{n}$ are positive. Permanent fluctuations (i.e., negative contributions) can turn the content of this buffer negative. Negative buffer indicates the presence of the "precursor oscillations". Tuning of $A_{c}$ is realized as $\dot{A}_{c}\left(t_{n}\right)=C_{ \pm} \operatorname{sign}\left(\hat{F}_{n}-F_{\text {min }}\right)$ with $F_{\min }=10^{-3}$ threshold and speed $C_{+}=3 \times 10^{-5}$ and $C_{-}=9 \times 10^{-5}$. The speed of decrease in $A_{c}$ is three times faster than that of its increase. The appropriate value for $\beta$ strongly depends on the cycle time of the controller and the smoothness of the trajectory to be tracked: its greater values allow the temporal occurrence of more shortterm fluctuations without reversing the time-development of the tuned $A_{c}$, therefore they are more robust regarding the external noises, too. For our purpose $\beta=0.5$ was satisfactory.

The simulations were implemented in SCILAB 5.4.0 and its XCOS graphical co-simulator. For numerical integration, the "Sundials/CVODE-BDF-FUNCTIONAL" option was chosen, with a step length freely determined by the simulator.

\section{B. Simulation results}

The first set of simulations was made for the upper initial position of the pendulum, i.e., for $q_{1_{\text {ini }}}=0$. In the graphs of trajectory tracking, the difference between the non-adaptive (without function $G$ ) and the adaptive (with function $G$ ) cases are invisible to the naked eye, therefore only this latter diagram is displayed in Fig. 3. On the other hand, Fig. 4 reveals that adaptivity reduces the tracking error. The chart of the generalized forces (see Fig. 5) well testifies the essence of the adaptive deformation. In the graph of non-adaptive case - when no adaptive deformation existonly two different lines can be seen since then $Q_{1}^{\text {Des }} \equiv$ $Q_{1}^{D e f}$. The realization of the MRAC illusion can better be observed in the zoomed excerpt of the adaptive force graph (Fig. 5): the "desired" and the "simulated" forces are in each other's close vicinity, and they are considerably different to the "adaptively deformed" ones. The appearance of the "precursor oscillations" is also evident observing the torque charts.

The tuned parameter $A_{c}$ versus time in the adaptive case is displayed in Fig. 6. It reveals that the cause of the observable torque fluctuations is the relatively high value of $A_{c}$, and the decrease in $A_{c}$ makes the small fluctuations cease.

The motion of the directly driven axle $q_{1}$ can be captured in Fig. 7. Similarly interpretable results are obtained for $q_{1_{\text {ini }}}=\pi$, i.e., for lower initial pendulum position and for the same nominal motion for $q_{2}$ (Figs. 9 and 10). In this case, the direction of the early displacement of $q_{1}$-as well as the sign of the necessary torque-are changed in a manner that is consistent with the qualitative picture of the physics 


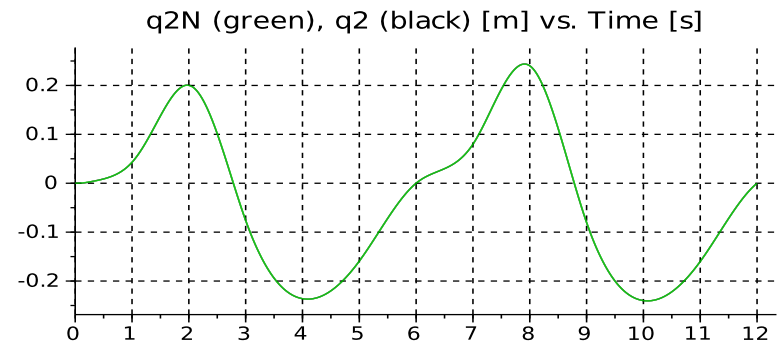

Fig. 3. Trajectory tracking for the adaptive case: $q_{2}$ simulated: black, $q_{2}^{\text {Nom }}$ : green lines (they are barely distinctive).

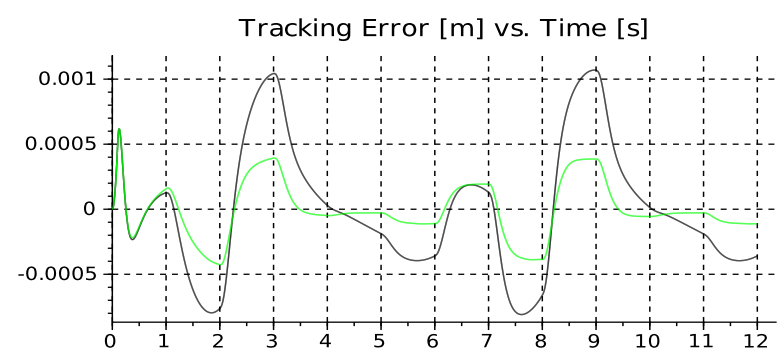

Fig. 4. Trajectory tracking error versus time for $q_{2}$ for the non-adaptive (black) and the adaptive (green) cases.

Q1: Des.(black), Def.(blue), Sim.(red) [Nm] vs. Time [s]

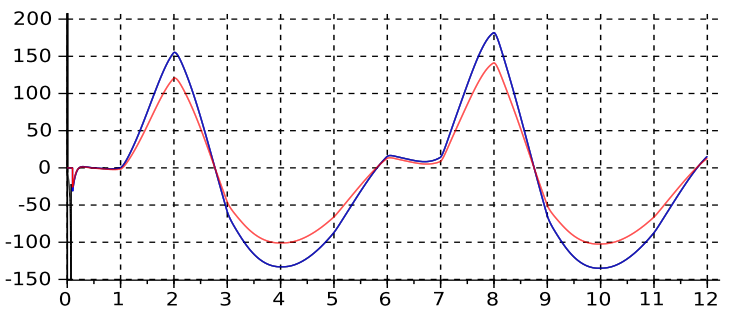

Q1: Des.(black), Def.(blue), Sim.(red) [Nm] vs. Time [s]

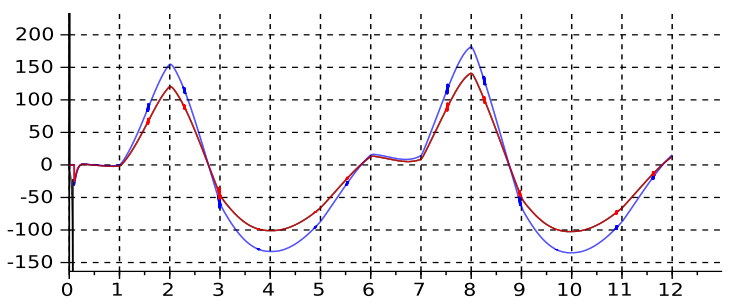

Q1: Des.(black), Def.(blue), Sim.(red) [Nm] vs. Time [s]

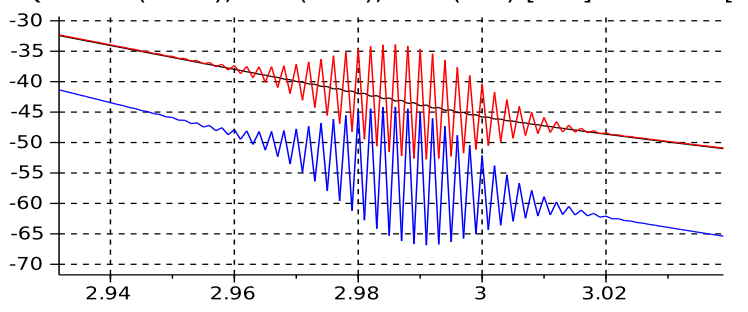

Fig. 5. The torque for the non-adaptive (upper) and the adaptive (middle and lower) cases versus time: $Q_{1}^{\text {Des }}$ : black, $Q_{1}^{\text {Def }}$ : blue, $Q_{1}^{\text {Sim }}$ : red lines. In the upper figure, since no deformation is applied, black line is identical with the blue one. On the other hand, in the middle figure, black line approaches the red one which corresponds to the "MRAC illusion". In the lower figure, a precursor oscillation can be seen which is relaxed rapidly (in $5 \mathrm{~ms}$ ).

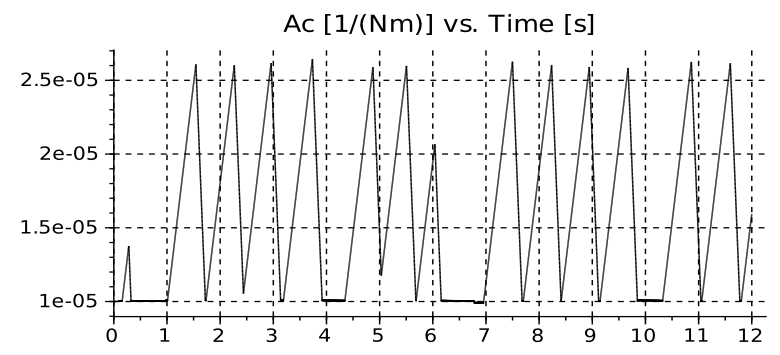

Fig. 6. The tuned adaptive parameter $A_{c}$ versus time.

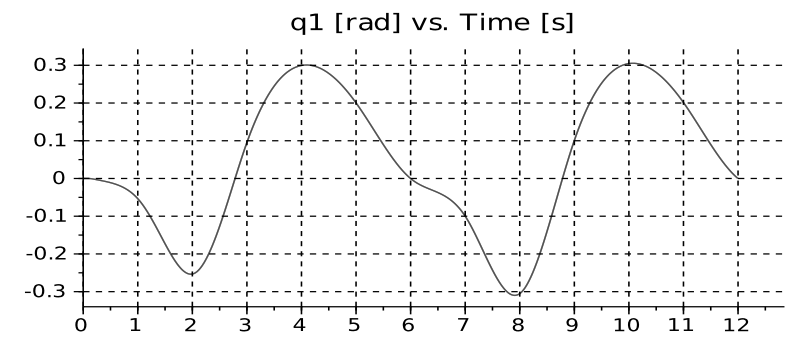

Fig. 7. The motion of the directly driven axle $q_{1}$ versus time for the adaptive control when $q_{1_{i n i}}=0$.

ddot q_2: Nom.(black), PID corr. (green) [m/s^2] vs. Time [s]

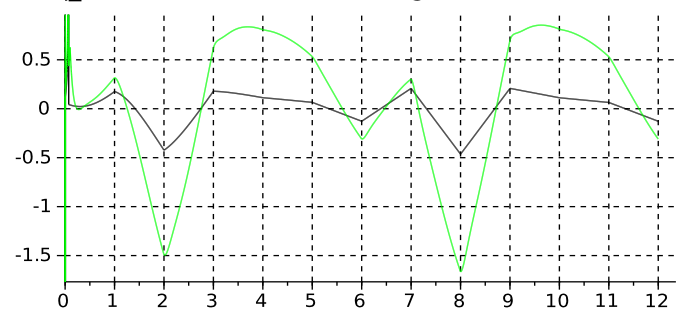

Fig. 8. The second time-derivatives: $\ddot{q}_{2}^{N}$ (black line) and the PID corrected one (green line) versus time for the non-adaptive control when $q_{1_{\text {ini }}}=0$.

of the system, i.e., with the varying sign of the system's "inertia term" in (8). Eq. (5) is determined according to the convention that the positive direction along axle $q_{2}$ is from left to right, while the positive rotational direction for measuring $q_{1}$ and $Q_{1}$ is defined clockwise.

For the comparison with more traditional (linear) control approaches, an example is shown for constant nominal trajectory $q_{2}^{\text {Nom }}=0.6$ where e.g. the setting time may be of interest. The results with initial conditions $q_{1_{i n i}}=0.4$ and $q_{2_{\text {ini }}}=0$ can be seen in Fig. 11 .

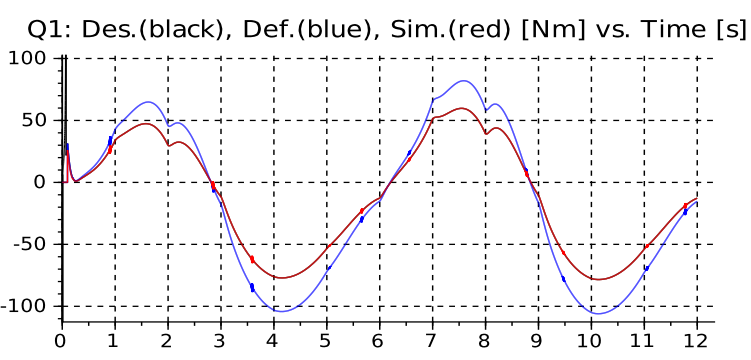

Fig. 9. The torque for the adaptive case versus time when $q_{1_{\text {ini }}}=\pi$ : $Q_{1}^{\text {Des}}$ : black, $Q_{1}^{\text {Def }}$ : blue, $Q_{1}^{\text {Sim }}$ : red lines. 


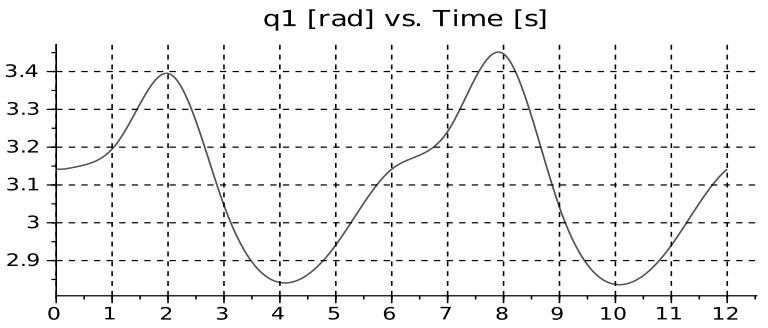

Fig. 10. The motion of axle $q_{1}$ versus time when $q_{1_{i n i}}=\pi$.
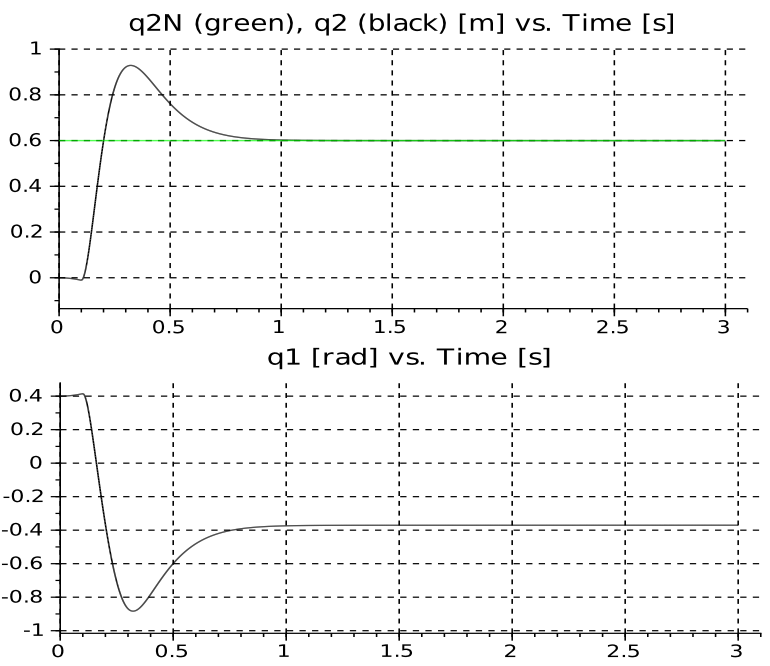

Fig. 11. Tracking a constant nominal trajectory in the adaptive case.

\section{CONCLUSions}

In control engineering, benchmark problems can reveal the strengths and weaknesses of the designed controllers in typical situations, and making them comparable to prior solutions. In this paper, an RFPT-based MRAC controller was designed for a modified TORA system. This is an indirectly driven, underactuated classical mechanical system that normally have dynamic singularities. These singularities correspond to the essential physical limits of the actuation, therefore their limits cannot be overridden by aggressive adaptive design. It is substantiated by simulations that the RFPT-based design of MRAC controllers can work well in adaptive control. Instead of applying the commonly employed Lyapunov's $2^{\text {nd }}$ method, it was shown that for the tuning of only one of the altogether three adaptive parameters of the control method, a simple rule-base can be developed. This makes the design method very efficient. On the basis of this rule-base, more intelligent control solutions can be developed that can use model-independent observers for parameter tuning. In the future, we wish to extend our investigations for underactuated systems having higher DoF, and support the results by physical experiments.

\section{ACKNOWLEDGMENT}

The authors thankfully acknowledge the grant provided by the Project TÁMOP-4.2.2.A-11/1/KONV-2012-0012: Basic research for the development of hybrid and electric
vehicles-The Project is supported by the Hungarian Government and co-financed by the European Social Fund. T. Haidegger and L. Kovács are Bolyai Fellows of the Hungarian Academy of Sciences.

\section{REFERENCES}

[1] U. Hartmann. Midcourse guidance techniques for advanced tactical missile systems. AGARD Lecture Series: Missile Interceptor Guidance System Technology (ADA228936), (173):27-53, 1990.

[2] M.F. Senning. Hydraulic positioning system. Davison, E.J. (Hrsg.); IFAC (Veranst.): Benchmark problems for control system design IFAC, 30-31:30-31, 1990.

[3] R. H. Cannon Jr. Dynamics of Physical Systems. McGraw-Hill, 1967.

[4] R.T. Bupp, D.S Bernstein, and V.T. Coppola. A benchmark problem for nonlinear control design: Problem statement, experiment testbed and passive nonlinear compensation. Proc. of the American Control Conference, Seattle, US, pages 4363-4376, 1995.

[5] Authors of a special issue. A special issue presenting 9 papers on controlling the TORA system. International Journal of Robust and Nonlinear Control, 8:305-457, 1998.

[6] M. Jankovic, D. Fontanie, and P.V. Kokotovic. TORA example: Cascade- and passivity based control designs. IEEE Transaction on Control System Technologies, 4:292-297, 2006.

[7] P. Baranyi, Z. Petres, P. Várlaki, and P. Michelberger. Observer and control law design to the TORA system via TPDC framework. WSEAS Transactions on Systems, 1(5):156-163, 2006.

[8] Jean-Jacques E. Slotine and W. Li. Applied Nonlinear Control. Prentice Hall International, Inc., Englewood Cliffs, New Jersey, 1991.

[9] R. Isermann, K.H. Lachmann, and D. Matko. Adaptive Control Systems. Prentice-Hall, New York DC, USA, 1992.

[10] C.J. Khoh and K.K. Tan. Adaptive robust control for servo manipulators. Neural Comput \& Applic, 12:178-184, 2005.

[11] R.M. Murray, Z. Li, and S.S. Sastry. A mathematical introduction to robotic manipulation. CRC Press, New York, 1994.

[12] C.C. Nguyen, S.S. Antrazi, Z-L. Zhou, and C.E. Campbell Jr. Adaptive control of a stewart platform-based manipulator. Journal of Robotic Systems, 10(5):657-687, 1993.

[13] R. Kamnik, D. Matko, and T. Bajd. Application of Model Reference Adaptive Control to Industrial Robot Impedance Control. Journal of Intelligent and Robotic Systems, 22:153-163, 1998.

[14] J. Somló, B. Lantos, and P.T. Cát. Advanced robot control. Akadémiai Kiadó, Budapest, 2002.

[15] K. Hosseini-Suny, H. Momeni, and F. Janabi-Sharifi. Model Reference Adaptive Control design for a teleoperation system with output prediction. J Intell Robot Syst, DOI 10.1007/s10846-010-9400-4:1-21, 2010.

[16] L. Kovács. Modern robust control in patophysiology from theory to application. IEEE 11th International Symposium on Applied Machine Intelligence and Informatics (SAMI 2013), page 13, 2013.

[17] J.K. Tar, J.F. Bitó, and I.J. Rudas. Replacement of Lyapunov's direct method in model reference adaptive control with robust fixed point transformations. 14th IEEE International Conference on Intelligent Engineering Systems 2010, Las Palmas of Gran Canaria, Spain, pages 231-235, 2010.

[18] S. Banach. Sur les opérations dans les ensembles abstraits et leur application aux équations intégrales (About the Operations in the Abstract Sets and Their Application to Integral Equations). Fund. Math., 3:133-181, 1922

[19] T.A. Várkonyi, J.K. Tar, I.J. Rudas, and I. Krómer. VS-type stabilization of MRAC controllers using robust fixed point transformations. In: Proc of the 7th IEEE International Symposium on Applied Computational Intelligence and Informatics (SACI 2012), May 24-26, 2012, Timişoara, Romania, pages 389-394, 2012.

[20] K. Kósi, Sz. Hajdu, J.F. Bitó, and J.K. Tar. Chaos formation and reduction in Robust Fixed Point Transformations based adaptive control. 4th IEEE International Conference on Nonlinear Science and Complexity (NSC 2012), Budapest, Hungary, pages 211-216, 2012.

[21] K. Kósi, J.K. Tar, and I.J. Rudas. Improvement of the stability of RFPT-based adaptive controllers by observing "precursor oscillations". In the Proc. of the 9th IEEE International Conference on Computational Cybernetics, 8-10 July 2013, Tihany, Hungary, pages 267-272, 2013 